Original Article

\title{
The effects of visual feedback balance training on the pain and physical function of patients with chronic degenerative knee arthritis
}

\author{
Hyunju Oh, PhD, $\mathrm{PT}^{1)}$, Sangyong Lee, PhD, $\mathrm{PT}^{2}$, Kwansub Lee, PhD, $\mathrm{PT}^{3)}$, \\ JiOUn CHOI, $\left.\mathrm{PhD}, \mathrm{PT}^{4}\right)^{*}$ \\ 1) Department of Physical Therapy, Taegu Science University, Republic of Korea \\ 2) Department of Physical Therapy, U1 University, Republic of Korea \\ 3) Department of Physical Therapy, Daegu Health College, Republic of Korea \\ 4) Department of Physical Therapy, Bullo Union Orhtopedics: 153 Palgong-ro, Dong-gu, Daegu 41034, \\ Republic of Korea
}

\begin{abstract}
Purpose] This study aimed to investigate the effects of visual feedback balance training on the pain and dysfunction of patients with chronic degenerative knee arthritis. [Participants and Methods] Twenty-six patients with chronic degenerative knee arthritis participated in this study; the control group $(n=13)$ performed muscle strength training and the experimental group $(n=13)$ performed visual feedback balance training. General physical therapy was applied to both groups three times a week for eight weeks. The visual analog scale was used to measure the patient's pain scale, and the K-WOMAC (Korean Western Ontario and McMaster Universities Osteoarthritis Index) was used as a tool to evaluate their physical function. [Results] In the intra-group comparisons, significant decreases in the visual analog scale and the K-WOMAC were observed for the control group and the experimental group. No significant difference was found in the inter-group comparisons after treatment. [Conclusion] Visual feedback balance training is considered to be an effective intervention method for improving pain and dysfunction in patients with chronic degenerative knee arthritis.

Key words: Visual feedback balance training, Pain, Chronic degenerative knee arthritis
\end{abstract}

(This article was submitted Apr. 21, 2020, and was accepted Jun. 3, 2020)

\section{INTRODUCTION}

Degenerative arthritis is a disease caused by wear and degeneration of the cartilage tissues that cushion the ends of bones and prevent friction; it is also called osteoarthritis or arthritis deformans. Due to the pain, stiffness, and reduced mobility range of the joint, the patient ultimately loses their functional independencel), and this condition is known to be highly related to muscle weakness of the quadriceps femoris muscle ${ }^{2}$. Degenerative arthritis can be prevented by regular exercise therapy while maintaining a healthy lifestyle, and even patients with arthritis can improve their disease condition by musclestrengthening and aerobic exercises ${ }^{3}$. Therefore, a pyramidal approach is usually applied to patients with degenerative arthritis; first, muscle-strengthening exercise of the quadriceps femoris muscle is recommended, followed by conservative treatment. If there is no improvement after the conservative treatment, drugs can be prescribed, starting with those with fewer side effects, such as acetaminophen and anti-inflammatory analgesic, and then, if this pharmaceutical therapy does not work, surgical intervention can be employed ${ }^{4)}$. Visual feedback training, which has been widely used recently, integrates afferent information from vestibular and somatic senses, contributing to posture control and reducing postural disturbance ${ }^{5)}$, so it is applied to improve postural balance and visual perception in patients with damage in the central nervous system or orthopedic

*Corresponding author. Jioun Choi (E-mail: ptchoi2001@hanmail.net)

(C2020 The Society of Physical Therapy Science. Published by IPEC Inc.

(c) (i) $\odot$ This is an open-access article distributed under the terms of the Creative Commons Attribution Non-Commercial No Deriva-

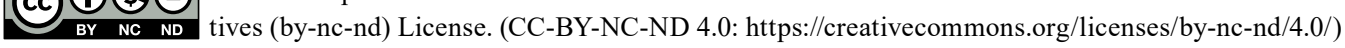


injuries. The primary goal in the treatment of patients with chronic degenerative knee arthritis is to reduce pain and improve gait and function. Laufer et al. ${ }^{6}$ ) reported that the visual-feedback effect played an important role in the postural control of patients with balance control disorders. The study of Hatzitaki et al. ${ }^{7)}$ in which a postural control experiment was conducted in elderly women, reported that the posture control ability of the participants was improved after a visual biofeedback mechanism was applied. Although the visual feedback balance training is widely used for various chronic degenerative knee arthritis, the evidence of their effects has not been firmly established.

Therefore, this study aimed to apply proprioceptive stimulation training accompanied by visual information in order to investigate effects on the pain and function of patients with chronic degenerative knee arthritis.

\section{PARTICIPANTS AND METHODS}

This study was conducted in patients of sixty years of age or older who were diagnosed with degenerative knee arthritis by an orthopedic specialist. The study group consisted of those hospitalized at G Hospital in Gumi, Gyeongsangbuk-do, South Korea. Those with any neurological diseases that may affect balance or gait and those with a past history of orthopedic surgery related to knee arthritis were excluded from the study. Consequently, twenty-six patients (males: 7, females: 19) were included in this study.

For the control group ( $\mathrm{n}=13)$, the mean age was $67.2 \pm 5.3$ years, the mean height was $158.8 \pm 7.6 \mathrm{~cm}$, the mean weight was $62.1 \pm 8.2 \mathrm{~kg}$; for the experimental group $(\mathrm{n}=13)$, the mean age was $64.5 \pm 3.6$ years old, the mean height was $160.0 \pm$ $8.4 \mathrm{~cm}$, and the mean weight was $60.9 \pm 13.6 \mathrm{~kg}$. Ethical approval for the study was granted by the U1 University Institutional Review Board (U1IRB2020-02). All participants read and signed consent forms in accordance with the ethical standards of the Declaration of Helsinki.

The control group performed a muscle-strengthening exercise using an elastic band (Hygenic Corporation, USA). To avoid straining muscles and joints, stretching and simple freehand exercises were performed in a comfortable state for warming up and cooling down. The warm-up and cool-down exercises were performed for five minutes, each mainly focusing on the flexibility of the large joints of the body. The exercise program was composed of three sets of 10 repetitions at maximum extension with a two-minute resting time after each set; this exercise took about 20 minutes total. The frequency of exercise was set to 10 times per set, and the participants were instructed to perform the exercise within the range of joint movement in which the participant's rating scales of perceived exertion were at intensity levels of 13 to 15 (a little hard to hard), which are the final levels of exertion before the subject begins to feel pain ${ }^{8)}$.

The experimental group performed visual feedback balance training in which Balance Trainer (Media Medizintechnik $\mathrm{GmbH}$, Germany) was utilized to create three simple games. The training program instructed participants to: move a rabbit from the center of the screen to an apple on the monitor, which could appear from eight possible directions, and then put the apple back into a basket in the center of the screen; touch pillars at corners in four directions while drawing circles on a donut-shaped screen; and hit a block-shaped box with a ball in a game similar to tennis. Both the control group and the experimental group were treated with general conservative physical therapy, during which hot and wet therapy (20 minutes), interference current therapy (100 bps, 15 minutes), and ultrasonic therapy ( $1 \mathrm{MHz}, 1.5 \mathrm{~W} ; \mathrm{cm}^{2}, 5$ minutes) were applied.

The Visual analogue scale (VAS) was used to evaluate the pain scale. The K-WOMAC (Korean Western Ontario and McMaster Universities Osteoarthritis Index) was used to evaluate physical function. The WOMAC is an index that evaluates the pain and functional status of patients with degenerative knee arthritis and is widely used as a measure for evaluating symptoms and treatment effects in patients with degenerative knee joints.

For the statistical processing in this study, a paired t-test was performed in the intra-group comparisons to determine the pain scale and the physical function of patients with chronic degenerative knee arthritis, and a two-sample t-test was performed in the inter-group comparisons after treatment. Data obtained from this study were statistically processed using Microsoft Excel 365 (Microsoft office Inc., Korea) and the significance level $\alpha$ was set to 0.05 .

\section{RESULTS}

The results of this study showed that in the intra-group comparisons, significant decreases in the VAS and K-WOMAC were observed for the control group and the experimental group $(\mathrm{p}<0.05)$. No significant difference was found in the intergroup comparisons after treatment $(\mathrm{p}>0.05)$ (Table 1).

\section{DISCUSSION}

Recently, studies have been actively conducted on the types of exercise therapies and programs that can induce joint movement without exerting excessive load on the articular cartilage affected by degenerative arthritis. Among these, visual feedback balance training has been widely represented ${ }^{9)} . \mathrm{Kim}^{10)}$ applied a knee joint muscle-strengthening exercise using an elastic band to determine the effect of such training on the muscle strength and balance control ability of the elderly, and it was found that both strength and balance, which reflect lower extremity strength, were enhanced after the five-week exercise; it was reported that this exercise also had a significant effect on improving the agility and dynamic balance control of the 
Table 1. Comparison of VAS and K-WOMAC within each group

\begin{tabular}{llcc}
\hline & Group & Pre-test & Post-test \\
\hline VAS $(\mathrm{cm})$ & Control group & $7.0 \pm 2.0$ & $4.9 \pm 1.4^{* *}$ \\
& Experimental group & $6.9 \pm 1.8$ & $4.2 \pm 1.7^{* *, !}$ \\
K-WOMAC (points) & Control group & $28.5 \pm 10.6$ & $22.9 \pm 8.3^{* *}$ \\
& Experimental group & $33.3 \pm 13.7$ & $15.7 \pm 8.7^{* *}, !$ \\
\hline
\end{tabular}

VAS: Visual Analogue Scale; K-WOMAC: Korean Western Ontario and McMaster Universities Osteoarthritis Index.

${ }^{* *}$ paired t-test; ${ }^{* *} \mathrm{p}<0.01 ;$ 'No significant difference between two groups, $\mathrm{p}>0.05$.

elderly. The study of Loughlin and Redfern ${ }^{5)}$ reported that all groups that underwent visual feedback showed an improvement in their short-term motor learning ability. The study of Hatzitaki et al. ${ }^{7)}$ in which a postural control experiment was conducted in elderly women, reported that the posture control ability of the participants was improved after a visual biofeedback mechanism was applied. Lim ${ }^{11}$ ) researched exercise paired with visual information in patients with degenerative arthritis and found a significant decrease in pain as measured using the visual analogue scale.

The results of this study showed that there were statistically significant decreases in pain and functional changes in both the control and experimental groups, but there was no significant difference in the inter-group comparisons after treatment. These results indicate that the visual feedback balance training reduced disturbance to the head and the trunk while performing the exercise and also reduced disturbance to the pelvis and the lower limbs. In addition, it is believed that the strategic movement of the knee joint was reduced due to the decreased disturbance to the body, which kept knee joint movement from the pain-inducing range ${ }^{12)}$. Further, as a result of the increase in muscle strength around the knee joint due to the musclestrengthening exercise, standing stability increased, which is likely a result of the improvement of functional ability due to pain relief.

Taken together, this study shows that knee joint muscle-strengthening exercise using visual information feedback training was effective in improving the balance ability and pain of patients with degenerative knee arthritis. This training method is expected to help patients with degenerative knee arthritis improve their standing balance ability and reduce pain.

\section{Conflict of interest}

None.

\section{REFERENCES}

1) Davies CM, Guilak F, Weinberg JB, et al.: Reactive nitrogen and oxygen species in interleukin-1-mediated DNA damage associated with osteoarthritis. Osteoarthritis Cartilage, 2008, 16: 624-630. [Medline] [CrossRef]

2) O'Reilly SC, Jones A, Muir KR, et al.: Quadriceps weakness in knee osteoarthritis: the effect on pain and disability. Ann Rheum Dis, 1998 , 57: 588-594. [Medline] [CrossRef]

3) Eyigor S, Hepguler S, Capaci K: A comparison of muscle training methods in patients with knee osteoarthritis. Clin Rheumatol, 2004, 23: 109-115. [Medline] [CrossRef]

4) Creamer P, Lethbridge-Cejku M, Hochberg MC: Factors associated with functional impairment in symptomatic knee osteoarthritis. Rheumatology (Oxford), 2000, 39: 490-496. [Medline] [CrossRef]

5) Loughlin PJ, Redfern MS: Spectral characteristics of visually induced postural sway in healthy elderly and healthy young subjects. IEEE Trans Neural Syst Rehabil Eng, 2001, 9: 24-30. [Medline] [CrossRef]

6) Laufer Y, Sivan D, Schwarzmann R, et al.: Standing balance and functional recovery of patients with right and left hemiparesis in the early stages of rehabilitation. Neurorehabil Neural Repair, 2003, 17: 207-213. [Medline] [CrossRef]

7) Hatzitaki V, Voudouris D, Nikodelis T, et al.: Visual feedback training improves postural adjustments associated with moving obstacle avoidance in elderly women. Gait Posture, 2009, 29: 296-299. [Medline] [CrossRef]

8) Kisner C, Colby LA: Therapeutic exercise: foundations and techniques. Philadelphia: FA Davis, 2012.

9) Yi KO, Han HW, Lee YS, et al.: The effects of water exercise on pain and depression of female elderly degenerative arthritis. J Korean Soc Aerobic Exerc, 2002, 6: $105-118$

10) Kim HK: Effect of knee muscle power strengthening using Thera band on the balance control ability in the elderly. DanKook Unviversity, Dissertation of master's degree, 2003.

11) Lim KB: Balance and pain in the osteoarthritis patients after bio-feedback and strengthening exercises. Daegu University, Dissertation of master's degree, 2009.

12) Cappa $\mathrm{P}$, Patanè $\mathrm{F}$, Rossi $\mathrm{S}$, et al.: Effect of changing visual condition and frequency of horizontal oscillations on postural balance of standing healthy subjects. Gait Posture, 2008, 28: 615-626. [Medline] [CrossRef] 